\title{
MODELACIÓN MATEMÁTICA EN CONTABILIDAD
}

\section{MATHEMATICAL MODELING IN ACCOUNTING}

\author{
Eliseo Ramírez Rincón ${ }^{1}$ \\ Universidad Libre de Colombia
}

\section{RESUMEN}

Como resultado de la investigación La variable, núcleo del pensamiento variacional en la modelación contable, se encontró desde un estudio eminentemente matemático que las cuentas de activos, pasivos y patrimonio neto como enfoque de análisis de un balance general pueden tener un comportamiento variacional, que además benefician a otros procesos contables como los de medición, representación, valoración y evaluación.

PALABRAS CLAVE: Modelación matemática, Modelación contable, variables, proceso de variación.

1 Profesor investigador en Educación matemática en las líneas de; modelación matemática como apoyo a otras disciplinas y lenguaje matemático; en la facultad de Ciencias Económicas, Administrativas y Contables de la universidad Libre, sede Bogotá Colombia. correo: eliseo. ramirezr@unilibre.edu.co

http://www.orcid.org/0000-0002-9885-5714

https://scholar.google.es/scholar?hl=es\&as sdt=0\%2C5\&q= Eliseo+Ramirez+Rinc\%C3\%B3n\&btnG=

CvLAC: $\quad$ http://scienti.colciencias.gov.co:8081/cvlac/visualizador/generarCurriculoCv.do?cod_rh=0000285382-

\section{RESUMO}

Como resultado da investigação $A$ variável, núcleo do pensamento variacional na modelação contábil, encontrou-se desde um estudo eminentemente matemático que as contas de ativos, passivos e património neto como enfoque de análise de um balanço geral podem ter um comportamento variacional, que ademais beneficiam a outros processos contábeis como os de medida, representação, valoração $e$ avaliação.

PALAVRAS-CHAVE: Modelagem matemática, modelagem contábil, variáveis, processo de variação.

\section{ABSTRACT}

As result of the research the variable, core of variational thinking in the accounting modeling, was an eminently study from an analysis mathematical study that the accounts of assets, liabilities and heritage net accounts as a 
general balance analysis approach can have a variational behavior, which also benefit to other accounting processes such as measurement, representation, assessment and evaluation.

KEY WORDS: Mathematical modeling, accounting modeling, variables, variation process.

\section{INTRODUCCIÓN}

Dada la importancia de la modelación contable como uno de los procesos importantes de la Contabilidad, en la investigación La variable núcleo del pensamiento variacional en la modelación contable, se partió de la siguiente pregunta enfocada desde las matemáticas: ¿Las cuentas en contabilidad pueden tener comportamientos de variable?, de ser así, entonces existen también procesos de variación contable entre cuentas a partir de lo cual se afectan otros procesos como los de medición, representación, valoración y evaluación. La investigación se apoyó en nociones topológicas entre ellas la de distancia, métrica y norma para revisar y describir las cuentas del balance general con enfoque en los activos, pasivos y patrimonio neto que permitiera generar modelos matemáticos que se articularan con el proceso contable en cuestión; revisando para ello algunas métricas posibles, que respondan fundamentalmente a situaciones contables de variación como la descrita en este artículo, esto de acuerdo con la problemática expuesta en la investigación mencionaday, además, teniendo en cuenta lo hecho hasta ahora por investigadores como García Casella en la modelación contable axiomática y por Mattessich en la modelación contable normativa y axiomática, así como también por la preocupación manifiesta de varios investigadores contables reconocidos, en torno a la importancia por el desarrollo de la axiomática contable.

La investigación realizada como referente de este artículo, responde además a resultados de otra investigación terminada en 2016 llamada "La Contametría multi dimensional" ${ }^{2}$ en la que se evidenció la necesidad e importancia de proponer modelos contables cuantitativos que resignificaran el propuesto por Luca Pacioli en el s. XV sobre la ecuación contable clásica, porque según críticas de los investigadores mencionados así como de otros en la consideración de que la partida doble no es suficiente para resolver situaciones propias del s. XXI. En este sentido en la investigación realizada sobre Contametría se evidenció que la ecuación de partida doble de Pacioli, desde un análisis matemático que incluyó las relaciones entre las variables mencionadas, responde a un caso particular en el que las cuentas son constantes y por lo tanto solo mide algunas situaciones particulares en un instante dado.

\section{PLANTEAMIENTO DE LA PROBLEMÁTICA DE INVESTIGACIÓN}

Tua Pereda, dice ${ }^{3}$ que él y algunos investigadores contables como Mattessich (1958, 1964-2002), García Casella (1997 a y b; 2001, 2002, 2005), ljiri (1967) consideran que las matemáticas y la Contabilidad han llevado caminos paralelos, y que en particular las matemáticas han proporcionado estructuras a la contabilidad que la han beneficiado como la de la ecuación de la partida doble de Pacioli, sin embargo, son enfáticos también en afirmar que desde hace años la contabilidad está estancada, particularmente en la modelación contable ${ }^{4}$, además, Tua menciona la importancia de estudiar el "posible carácter predictivo" de la contabilidad, "la contabilidad enedimensional frente a la tradicional partida doble", entre otros

2 Desarrollada por el grupo de investigación Constructores Contables cuyo aporte se hizo desde la modelación matemática como aporte a la modelación contable cuantitativa.

$3 \quad$ Las fechas fueron puestas por mí y corresponden a trabajos relacionados en modelación contable cuantitativa por los autores mencionados por Tua Pereda.

$4 \quad$ La contabilidad es cuantitativa y la modelación contable cuantitativa, requiere de la modelación matemática. 
aspectos relevantes de la investigación en Contabilidad (Tua, 1988).

También se pueden mencionar otros investigadores para quienes la modelación matemática es importante en el desarrollo histórico de la contabilidad, tales como: Lopes de Sa (2002 a y b, 2003, 2007); Arenas (2004, mencionado por Ramírez, 2017); Cano (2008); Cano, Zapata y Ramírez (2005); Ramírez y Avellaneda (2017); García Serna (2011); Churruca (1982) quienes también han hecho aportes importantes en la modelación cuantitativa contable.

Un argumento fuerte para considerar la importancia de la modelación matemática como aporte a la modelación contable corresponde a una de las conclusiones que Jesús Arenas Herrera escribió en su trabajo La modelación matemática como base de la autonomía científica de la contabilidad (2004, mencionado en Ramírez, 2017), al respecto dice que debido a la escasa formación de los contadores en "métodos analíticos cuantitativos" se han generado dos situaciones consideradas por él como graves:

Deficiencias en el marco científico de la Contabilidad que la relegan de otras disciplinas.

$>$ El sometimiento a una limitada visión del ejercicio propio del Contador Público a la actividad de "llevar los libros", cuya consecuencia puede ser la "invasión" de su quehacer disciplinar en favor de profesionales de otras disciplinas. (Arenas, 2004, mencionado en Ramírez, 2017).

Dice Ramírez (2017) que Arenas, también llama la atención a la comunidad de contadores y a los investigadores contables para que establezcan conexiones con problemáticas propias de la naturaleza contable desde la axiomática, que permita construir teorías contables de índole científico, a partir de las cuales se responda por situaciones contables, como por ejemplo los procesos contables de variación, los cuales corresponden a una de las ramas de la naturaleza contable llamada modelación contable. Las situaciones de variación llevan inmersas interacciones entre variables cuya covariación se puede estudiar a partir de modelos matemáticos, que pueden ser generados por métricas matemáticas que dan sentido y orientan científicamente a la medición contable, como se verá en este trabajo particularmente en la ecuación de patrimonios.

En este sentido y a partir de la investigación "Contametría Multidimensional" (2016), realizada por dos grupos de investigación interdisciplinar, se propusieron elementos métricos con los que se construyeron modelos de medición patrimonial y quedaron preguntas como " ¿El contador debe ser sólo un agente de control? o está obligado a ir más allá, en la toma de decisiones, lo cual como dice Arenas en Ramírez (2017), lo obliga a refinar el procesamiento de la información que está a su cargo a partir de postulados científicos, que como en esta propuesta están centrados en la modelación matemática contable. En la investigación en cuestión se generó como producto un artículo científico publicado en 2016. En él se hace un análisis a la ecuación contable clásica de Pacioli, escrita en 1594 y vigente en nuestros días, casi que, como el único modelo matemático en los procesos de un balance general, porque en contabilidad circulan otros modelos matemáticos o mejor económicos, pero, que son prestados.

En el artículo se demostró, además, que esta ecuación de Pacioli es un caso particular de una ecuación contable general, la cual es entre otras de naturaleza vectorial y responde a situaciones patrimoniales diversas, inclusive en casos en los que se requieren patrimonios (de adición o pasivos que se adquieren para aumentar capital de trabajo en un momento 
dado) para garantizar la equivalencia entre los patrimonios neto $(\mathrm{Pn})$, de terceros (pasivos) y los de derechos (activos). Otro aporte importante del artículo de investigación fue el de estudiar situaciones contables que están por fuera del conjunto de los números reales, en cuyos casos debe recurrirse a un conjunto mayor que el de los números reales como lo es el conjunto de números complejos $(\mathrm{C}=a+b \mathrm{i})$ el cual contiene a los reales $(a, b)$ y a otros números llamados imaginarios $(i)$.

A partir de lo anterior y del interés particular heredado de la investigación mencionada "Contametría Multidimensional", se generó la necesidad de continuar con la línea de "investigación" axiomática en modelación contable cuantitativa, particularmente en la medición y modelación de cuentas desde una postura matemática, como proceso de variación; en la que se estudiará el cambio generado por variables contables de naturaleza cuantitativa en escalas de razón y de intervalos, es decir en las que se asume la existencia del cero como un elemento de unidad de medida.

\section{DESCRIPCIÓN DEL PROBLEMA DE INVESTIGACIÓN}

La contabilidad ha logrado desarrollar procesos de medición contable, básicamente a partir del uso de las escalas Nominal y Ordinal, porque no requieren del cero como número de comparación o patrón de medida, además, porque los escasos desarrollos en modelación cuantitativa como parte de la axiomática contable le han impedido hacerlo en las escalas de razón y de intervalos, para las que el uso y la existencia del cero es fundamental como patrón o unidad de medida.

Otro aspecto relevante para este trabajo investigativo fue la consideración hecha por algunos expertos en investigación contable mencionados antes, en torno a la limitación de la ecuación de partida doble de Pacioli. En este sentido, y dado que los grupos de investigación en los que se desarrolló la investigación reportada, han venido generando aportes investigativos a la modelación contable cuantitativa desde las matemáticas, particularmente a la ecuación de patrimonios, que han dejado interrogantes como el que se trabajó en esta investigación sobre la posible naturaleza variacional de un balance general, lo cual permitiría avanzar en la medición contable desde modelos matemáticos centrados en procesos variacionales como el cálculo diferencial e integral que requirieran del uso de las escalas de intervalos y de razón antes mencionadas.

Además de lo anterior, tanto en la problemática, como en los antecedentes de la investigación se encontró particularmente que sobre modelación contable cuantitativa hay especial interés, pero pocos desarrollos desde la postura axiomática en procesos de variación contable y además aclarando que teóricamente en la investigación se reportó que el comportamiento de las cuentas de un balance general con enfoque en los activos, pasivos y patrimonio neto es de naturaleza variacional, se propuso la siguiente pregunta.

\subsection{PREGUNTA DE INVESTIGACIÓN.}

¿Cómo modelar matemáticamente un balance general como un proceso de variación contable?

\subsection{JUSTIFICACIÓN}

La investigación La variable núcleo del pensamiento variacional en la modelación contable se propuso como continuación de los desarrollos alcanzados en la investigación Contametría multidimensional, realizada 
en la facultad de Ciencias Económicas, Administrativas y Contables de la universidad, en la que se estableció que la contabilidad como disciplina axiomática, debe cuestionar su naturaleza, objetos de estudio y de otra parte hacerse cargo de su propio proceso de medición contable; ya sea a través de métricas matemáticas o de métricas cualitativas apoyadas en índices e indicadores. En la investigación referida se planteó la pregunta de investigación ¿Existe una región de fenómenos contables en la que no se cumpla la ecuación contable clásica?, con dos objetivos generales: 1. Demostrar que la relación de patrimonios puede ser de equivalencia o de orden y 2. Demostrar que toda relación patrimonial es vectorial. A partir de la formulación de los objetivos, se realizaron las siguientes acciones para poder dar solución a la pregunta de investigación.

a. Se hizo un análisis de las características, propiedades y relaciones de cada una de las variables patrimoniales, teniendo en cuenta la ecuación contable clásica $(P=A-P a)$, para la que $P$ : patrimonio neto, $A$ : patrimonio de derechos y Pa: patrimonio de terceros (pasivos).

b. Se evaluaron algunos fenómenos patrimoniales que ocurren fuera del sistema determinado por la ecuación contable clásica y a partir de lo encontrado,

c. Se formuló un modelo para expresar y/o representar estos fenómenos de ocurrencia diferente a los que conforman la ecuación contable clásica, con lo que,

d. Se propuso un replanteamiento para la ecuación contable clásica, como relación de orden y como relación de equivalencia.

e. Se encontró que en la contabilidad axiomática es fundamental la modelación matemática, para poder generar la modelación contable en procesos de medición contable cuantitativa.
De otra parte, se justificó la investigación que generó este escrito porque el pensamiento variacional hace parte del marco legal emanado del MEN dirigido a estudiantes de la Básica (6-15 años) y Media (15-16 años) a través de los Lineamientos Curriculares (1998) y los Estándares Básicos de Competencias (2003 y 2006), en donde se puede inferir según los Lineamientos Curriculares que los contenidos curriculares del área de matemáticas se reorganizan en cinco pensamientos, entre los que se encuentra el pensamiento variacional conformado por los sistemas algebraicos y analíticos, mismos que tradicionalmente se proponen a partir octavo (13 y 15 años) hasta once (15-18 años). En los Estándares, se propone que los pensamientos y procesos generales sean transversales y horizontales desde el grado $1^{\circ}$ (6-7 años) hasta el grado $11^{\circ}$ (16-17 años), sin embargo el pensamiento variacional por su importancia en los fenómenos naturales, sociales y humanos tiene un particular interés tanto a nivel nacional como internacional en las pruebas estandarizadas (PISA, SABER, TIMMS, ...), mismas que según reportes de OCDE como del MEN dicen que los resultados de los estudiantes colombianos y latinoamericanos en general son deficientes. Además, en los mismos documentos se establece como uno de los procesos generales a la modelación matemática en diversos contextos (matemático, de otras ciencias, cotidiano, ...).

De otra parte, dado que la Contabilidad se puede entender como una ciencia social que se ocupa de diversos fenómenos sociales que le atañen y, como dice García Casella es limitante la afirmación que considera a la Contabilidad únicamente como aquella que tiene en cuenta los "hechos del pasado y del presente" (García, C., 2001). Se entiende desde los trabajos de él que la contabilidad debe ser predictiva $y$ apoyar en la toma de decisiones, y por lo tanto la modelación matemática como lenguaje científico es fundamental para estudiar, modelar 
y responder a algunos de los fenómenos de variación que surgen en las prácticas contables, como por ejemplo, en el balance general como una relación entre las cuentas de patrimonio, activos y pasivos; no desde la postura limitada de la aritmética y el álgebra elemental únicamente, sino trascendiéndolas al campo del análisis matemático (cálculo diferencial e integral), del álgebra lineal (vectores, matrices, grafos, ...), de la geometría analítica y fractal entre otras.

El pensamiento variacional tiene que ver con el tratamiento no solo matemático de la variación y el cambio en una situación, a través de las interacciones entre las variables que intervienen en el suceso o fenómeno; sino que también es importante entender en ella qué varía (el balance general), por qué varía (por la interacción entre las cuentas; activos, pasivos y patrimonio neto) y cómo varía (resultado de investigación). Esa fue la esencia del problema investigado, porque en Contaduría los estudiantes deberían enfrentarse a diversas situaciones de variación, que se les propongan en la carrera a través del currículo general para que, al graduarse de contadores les permita resolver situaciones nuevas en las que la variación está presente y para las que no fueron preparados en la universidad, no solo en la especificidad de la situación sino en el proceso de variación mismo también, porque al dejar de lado el estudio de los fenómenos variacionales se generan vacíos que aíslan a la comunidad de contadores de la formación útil y pertinente para enfrentarlas o asumirlas, quedando relegados a las implicaciones de seguir siendo profesionales del control desde el estudio de cuentas estáticas en el tiempo.

En este sentido, el pensamiento variacional que está ligado con la modelación matemática podría también estar en consenso con fenómenos propios de la contabilidad y esta característica, permitiría primero; la identificación de un fenómeno contable variacional, segundo; poder describir lo que sucede en él, detallando las variables que interactúan (covariación, qué varía, cómo lo hacen y por qué lo hacen) y tercero; intentar cuantificar o generar un patrón de regularidad (modelación matemática) que describa sucesos con características similares, que permitan predecir y anticipar situaciones propias de ésta en un fenómeno contable.

Otro aspecto que justifica la investigación corresponde a las exigencias hechas por la comunidad de investigadores contables liderada por Mattessich, Gomberg, ljiri, García Casella... sobre la partida doble, para quienes: "la partida doble convencional acusa deficiencias y críticas desde hace algunos años; una evidencia de ello es que diversos autores han realizado propuestas alternativas (causal, matricial, vectorial, ...) y han hecho críticas a ésta por sus deficiencias" (García, C. 2002). Al respecto García G. (1975) dice lo siguiente: “...todo esto supone la desaparición de la partida doble como tal y del monopolio que tenía como herramienta básica en la administración de negocios" (pág. 3), se evidencia la urgencia de que la contabilidad asuma una postura científica que permita a los contadores responder a situaciones no solo como control, sino que supere esta limitación que como barrera viene arrastrando desde el s. $\mathrm{XVI}$.

\section{OBJETIVO GENERAL DE LA INVESTIGACIÓN ${ }^{5}$}

Proponer métricas matemáticas que generen modelos cuantitativos en ámbitos de la Contabilidad en procesos de variación como aporte a la medición contable.

\section{OBJETIVOS ESPECÍFICOS}

4.1. Hacer una revisión documental sobre la modelación matemática en procesos de variación contable.

$5 \quad$ La variable núcleo del pensamiento variacional en la modelación contable 
4.2. Clasificar los desarrollos alcanzados por la modelación matemática en ámbitos de la contabilidad.

\subsection{Revisar situaciones contables de} fenómenos patrimoniales, cuyos procesos de variación generen modelos contables cuantitativos, a través de métricas matemáticas.

4.4. Proponer modelos con métricas matemáticas, para la medición de patrimonios en procesos de variación.

\section{METODOLOGÍA DE LA INVESTIGACIÓN}

La metodología propuesta para el trabajo de investigación fue de naturaleza cualitativa, tipo investigación acción educativa con enfoque descriptivo y analítico, que inició en el primer semestre de 2017 con cuatro fases de investigación; la primera correspondió a un proceso de revisión documental apoyado por estudiantes del semillero FRACTALES, a quienes se les pidió trabajar alrededor de temáticas pertinentes a la modelación matemática en las ciencias contables o de la Administración de Empresas desde la perspectiva de un balance general en un marco de investigación formativa. A ellos se les dijo que tomaran un período corto en la historia de la contabilidad para indagar por la axiomática contable o la relación con las matemáticas a partir de una revisión documental, además se diseñó y validó una grilla de categorías en la que se establecieron 10 categorías de análisis: autor (investigador, teórico,... ); época; desarrollo social y cultural; desarrollo económico; desarrollo epistemológico; desarrollo político; desarrollo tecnológico; desarrollo teórico; influencia en la contabilidad axiomática o la aplicación de modelos matemáticos. Las categorías de análisis debían ser descritas después de la revisión documental desde fuentes primarias y secundarias de diversa índole (investigaciones, libros, artículos científicos, ...) en un documento escrito, el cual fue revisado primero en el semillero y luego por pares externos para ser depurado.

La segunda fase correspondió a la clasificación de información según las categorías establecidas en la grilla, la importancia para la investigación fue la de organizar la información a partir de los trabajos realizados por los estudiantes del semillero cuyo logro fue alcanzado después de una discusión que generó acuerdos alrededor de la revisión documental hecha por ellos y ampliada en el trabajo de investigación.

La tercera fase fue de contrastación de información generada por las categorías de análisis y la cuarta fase de la investigación fue la de resultados, en la que se encontró la naturaleza variacional de las cuentas desde el punto de vista matemático, que permitió hacer la pregunta: ¿en contabilidad, hay casos prácticos en los que se de ese comportamiento variacional de un balance general?

Los desarrollos alcanzados por los diversos trabajos del semillero sobre modelación matemática en contabilidad han sido posibles a través de una investigación formativa enmarcada en una metodología de acción educativa, la cual se realizó como un fractal ${ }^{6}$ (replicas similares) que permitiera presentar, entender los avances y desarrollos en el uso de la modelación matemática desde trabajos particulares propuestos tanto en Administración de Empresas como en Contabilidad. De otra parte, en el grupo DIMATES hay especial interés por la modelación matemática que pueda contribuir en el desarrollo de la medición contable como uno de los ejes fundamentales de la contabilidad y que por lo tanto fue el foco de interés para el investigador principal y coordinador del semillero, al respecto, se propusieron métricas matemáticas en teoría de conjuntos y en cálculo (análisis matemático) que desbordan los modelos matemáticos usuales centrados en la

6 Las réplicas similares tienen que ver con la modelación matemática como eje articulador. 
aritmética y en el álgebra elemental, atendiendo a que el objetivo principal de la propuesta investigativa fue el uso y aprovechamiento de la modelación matemática en el desarrollo de modelos contables cuantitativos en procesos de variación y para ello se ubicó como situación o fenómeno variacional el balance general, por la importancia para determinar el estado financiero de una empresa o entidad en un momento dado.

\section{MARCO TEÓRICO DE REFERENCIA CONTABLE}

En este artículo se tomaron solo aquellos relacionados con el Balance General y con la modelación matemática en contabilidad. ${ }^{7}$

\subsection{MODELO CONTABLE}

Según Fowler, es posible considerar a un modelo contable como un esquema teórico que se propone para entender como ha sido el desarrollo evolutivo del patrimonio de las entidades (2011, p. 409). En el mismo sentido Álvarez (2010), tiene la misma percepción sobre la concepción de modelo contable, desde la cual es posible en un tiempo determinado, analizar cualquier situación patrimonial de una entidad que permita conocer la evolución patrimonial y así mismo anticipar sus comportamientos venideros. Según ellos, hay diferencias respecto a la manera como se entienden y trabajan las necesidades concretas de los usuarios, ya sean inversionistas, acreedores, empleados o clientes; porque la diferencia también determina, que el modelo contable permita adaptarse a las circunstancias particulares, de tal manera que la información recabada se corresponda con la decisión tomada para cada uno de los usuarios y que no sea la misma para todos o varios de ellos. De otra parte, también se encuentran propuestas de modelos contables con estructura axiomática como, por ejemplo, las de Balzer y Mattessich (1991) para quienes

$7 \quad$ En la investigación reportada se tuvieron en cuenta los trabajos particulares de los estudiantes que hacen parte del semillero de investigación. los modelos contables pueden ser modelados con ecuaciones simultáneas. En este mismo sentido, Bry y Casta (1995), y Beaver (1996) dicen que la información y la medición son conceptos fundamentales en la determinación de un modelo contable.

Así mismo, en la normatividad contable se encuentra la siguiente definición sobre lo que debe entenderse por valor razonable "...es el precio que se recibe al vender un activo o el que se paga al transferir un pasivo en una transacción ordenada entre participantes del mercado en la fecha de la medición." (NIIF 13, 2011), porque "el valor razonable" en la NIIF 13 está definido como una medición desde el mercado y no como una medición específica de una entidad.

\subsection{BALANCE GENERAL}

Se hace a partir de los saldos contables de las cuentas de activos, pasivos y patrimonios (capital de trabajo) los cuales se obtienen al finalizar un período contable o ejercicio (Romero, 2002, p.57). Existen dos formas diferentes de presentar un estado de situación financiera, la primera, se hace a través de un reporte que corresponde a la ecuación de; Patrimonio neto

= Activos - Pasivos y la segunda, a un balance en forma de cuenta, la cual corresponde a una variación de la ecuación de patrimonios, que destaca la relación que hay entre activos y pasivos; Activos $=$ Pasivos + Patrimonio neto . Los estados financieros son periódicos y deben permitir ser comparados entre ellos y con los de otras empresas del mismo sector (Kennedy \& McMullen, 1971; Ramírez, 1981; Romero, 2002).

El patrimonio neto ha sido objeto de investigaciones de índole financiero, económico y contable, al respecto se puede verificar en una tesis doctoral su importancia desde la década de los 90 , para la contabilidad, además porque establece consideraciones amplias respecto a la 
relación entre los activos y pasivos, como, por ejemplo, a mayor inversión asignada al capital será menor el valor correspondiente en los resultados y viceversa (Chaves, 1990).

"Es habitual, en nuestro país 8 , que las obras sobre contabilidad dediquen cuidadosa atención al examen de los aspectos vinculados con la medición y valuación de activos y pasivos, y consideren en cambio al patrimonio neto con mucha menor extensión y profundidad, a pesar de tratarse de uno de los temas que han merecido mayor regulación en la legislación positiva." (Chaves, 1990, pág. 8).

\subsubsection{ACTIVOS}

Con frecuencia se asumen como los recursos disponibles e inmediatos en dinero, inventarios, mercancías, títulos o cuentas por cobrar que forman parte de una entidad contable en un momento determinado y con los que es posible afrontar como garantía a las obligaciones propias, con el fin de asegurar su funcionamiento normal (Moreno, 2002; Rojas et-al, 1996).

Los activos pueden ser a corto plazo, también llamados circulantes o corrientes, que corresponden a las inversiones y recursos de una empresa o entidad contable que se pueden convertir en efectivo en un plazo no mayor a un año contable, con los cuales se puede pagar también a los acreedores dentro de ese tiempo y que permita a la empresa o entidad hacer frente a su situación para mantenerla en marcha con normalidad. De otra parte, están los activos a largo plazo, no circulantes o no corrientes; definidos como aquellos que se convertirán en efectivo en un tiempo mayor a un año contable y que, además es posible usarlos para el pago de deudas contraídas por una entidad económica también a largo plazo (Pyle, White, \& Larson, 1981; Moreno, 2002 y Prieto, 2009).

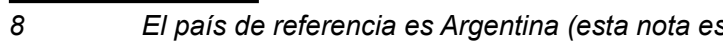
mía).
}

\subsubsection{PASIVOS}

Corresponden a la totalidad de aquellas obligaciones y deudas que han sido contraídas por una entidad contable ya sea en compras de mercancías a crédito o por pago de servicios como salarios y sueldos para los trabajadores o empleados; así como impuestos, hipotecas y documentos legales que la entidad está obligada legalmente a pagar en una fecha previamente fijada ya sea con bienes, dinero o servicios (Rojas et-al, 1996; Moreno, 2002).

Los pasivos también pueden ser a corto plazo, los cuales son conocidos como circulantes o corrientes y corresponden a las obligaciones contraídas como deudas o cuentas por pagar entre ellas sueldos, salarios, efectivo; que deben ser pagados dentro del período o ciclo contable de una entidad económica y que por lo tanto para ello debe hacer uso de los activos corrientes disponibles o en su defecto deberá crear otros pasivos circulantes (Pyle et-al, 1981; Rojas et-al 1996). Así mismo, están los pasivos no corrientes, no circulantes o a largo plazo que son obligaciones legales contraídas para ser pagadas después de un año del ciclo contable de la entidad deudora, que además podrán ser pagadas a través de los activos circulantes disponibles. De lo anterior, se deduce que dichas deudas son contraídas por una entidad económica con el fin de mejorar y beneficiarse con recursos económicos disponibles por terceros y que le pueden salir a un costo económico. Regularmente son usados para la compra de activos fijos, expandir la empresa o entidad, pagar deudas a corto o largo plazo. (Rojas et-al, 1996; Moreno, 2002).

\subsubsection{PATRIMONIO NETO O CAPITAL CONTABLE.}

Representa el interés de los propietarios de una empresa u organización, sobre las acciones que ellos tienen, que por lo tanto se hace necesario fijar en la entidad económica el porcentaje de 
activos que se están financiando con dichos recursos.

(Kennedy et-al, 1971; Pyle et-al, 1981). Los autores anteriores hacen referencia a que; el término capital contable es más usado por las entidades económicas con ánimo de lucro, mientras que el de patrimonio contable se asimila con entidades sin ánimo de lucro, sin embargo, dicen ellos también que ambas expresiones se refieren y representan lo mismo.

Las cuentas de activos, pasivos y patrimonio neto conforman el balance general de una entidad económica, para lo cual es importante que los activos sean clasificados de acuerdo con su disponibilidad, lo cual corresponde o representa la rapidez con la que ellos pueden convertirse en efectivo, en tanto que los pasivos se pueden presentar de acuerdo con las exigencias hechas a la entidad, las cuales varían de una entidad contable a otra, porque son dependientes de la importancia asignada a cada cuenta que conforme los pasivos; según (Kennedy et-al, 1971; Pyle et-al, 1981) por lo general se debe iniciar por poner a los proveedores, seguidos por los documentos e impuestos por pagar y dejando al final todos los demás deudores. En el mismo sentido ellos dicen, que las cuentas que conforman el capital, se deberán presentar en primer lugar el capital aportado por los socios, dueños o accionistas de la organización, empresa o entidad y luego el capital ganado a través de las operaciones propias de la entidad contable, así mismo si existieren pérdidas también es necesario indicarlas, (Hatzacorsian, 2003).

\subsection{LA INFORMACIÓN FINANCIERA Y LA TOMA DE DECISIONES.}

Es importante determinar y describir el estado de posición financiera de una entidad contable, para lo cual se hace indispensable revisar y analizar la información financiera disponible a través de herramientas como los indicadores financieros, con metodologías coherentes con las que sea posible determinar un proceso para la toma de decisiones confiables y seguras. Los indicadores financieros presentan las relaciones entre una cifra con otra en un estado financiero, que permiten evaluar los resultados de las operaciones de una entidad económica como, por ejemplo, la rentabilidad económica, la liquidez o el uso de activos y pasivos de una entidad (Romero, 2002; Guajardo y Andrade, 2008).

Entre los indicadores financieros que se aplican al Balance General, están los que hacen referencia a la liquidez de una entidad económica, en particular las que generan la razón del capital de trabajo o también conocida como la razón circulante $\left(R_{c}\right)$, la cual establece una relación entre los recursos financieros de una entidad económica en el corto plazo con el fin de hacerle frente a las obligaciones de pagos y que fueron contraídas en el mismo período contable; la finalidad es la de poder determinar la capacidad de pago de la entidad, para que resuelvan los compromisos de pago adquiridos. El resultado de la se expresa como el número de veces que a la entidad económica puede hacerlo, como se puede apreciar en la tabla1.

La razón circulante $\left(R_{C}\right)$ genera una de las siguientes tres posibilidades en una empresa o entidad contable.

Tabla 1. Razón circulante (el símbolo >>: se lee como mucho mayor).

\begin{tabular}{|l|l|l|}
\hline $\begin{array}{l}R_{C}: \text { Razón } \\
\text { circulante. }\end{array}$ & & $\begin{array}{l}R_{C}<1: \text { No hay } \\
\text { capacidad de asumir } \\
\text { compromiso de } \\
\text { pagos a terceros. }\end{array}$ \\
$\begin{array}{l}A_{C}: \text { Activo } \\
\text { circulante. }\end{array}$ & $R_{C}=\frac{A_{C}}{P_{C}}$ & $\begin{array}{l}R_{C}>1: \text { Se pueden } \\
\text { asumir compromisos } \\
\text { de pago a terceros. }\end{array}$ \\
$\begin{array}{l}P_{C}: \text { Pasivo } \\
\text { circulante. }\end{array}$ & & $\begin{array}{l}R_{C}>>1: \text { Puede que } \\
\text { haya capital ocioso. }\end{array}$ \\
\hline
\end{tabular}

Fuente: propia. 
Es importante mencionar de la tabla 1., que entre más grande sea el resultado de $R_{c}$ aumenta la probabilidad de pago de los pasivos de la entidad económica, sin embargo, también hay que tener cuidado porque si el resultado obtenido para $R_{c}$ en el número de veces que los activos contienen a los pasivos es muy alto, puede significar que la entidad económica tiene capital ocioso y que bien pudiera ser usado para la generación de inversiones nuevas que le signifiquen fuentes de nuevos posibles ingresos; así mismo si el resultado para es menor que uno (unidad), indica que la entidad económica no tiene la capacidad de afrontar las obligaciones contraídas en el corto plazo (Romero, 2002; Guajardo et-al, 2008).

De otra parte, es posible estudiar el comportamiento variacional de la razón circulante a partir de las cuentas de activos y pasivos que varíen en función del tiempo y por lo tanto también cambiará en función del tiempo.

En este sentido, es importante establecer condiciones propias para la ecuación de, de la tabla 1. En primer lugar, los pasivos no pueden ser cero, por obvias razones (no estaría definida ), en segundo lugar, las cuentas de activos y pasivos no deben ser constantes, porque la razón circulante marginal sería cero.

$$
R c(t)=\frac{A_{c}(t)}{P_{c}(t)} \quad[\text { ec. 1] }
$$

De la ec. 1 , se infiere que tanto la razón circulante como las cuentas de activos y pasivos son funciones del tiempo y por lo tanto, puede existir una razón circulante marginal que mida la velocidad de cambio de las cuentas de activos y pasivos circulantes en función del tiempo, sería útil en el sentido de poder predecir los cambios, conociendo los intervalos de crecimiento y decrecimiento de la razón circulante, así como su máximo y/o mínimo valor.
De lo anterior, atendiendo a la condición de funciones del tiempo, la ec. 1 se puede derivar como un cociente, dando lugar a la ec. 2.

$$
R_{C}^{\prime}=\frac{P_{C} A^{\prime} C-P^{\prime}{ }_{C} A_{C}}{P_{C} 2} \quad \text { [ec. 2] }
$$

La ec. 2, surge de la condición de periodicidad del ciclo contable y de la relación entre las cuentas (A y $\mathrm{P}$ ) cuyo comportamiento matemático corresponde a funciones del tiempo $[\mathrm{A}(\mathrm{t})$ y $\mathrm{P}(\mathrm{t})]$ que, por lo tanto, generan que la razón circulante sea también una función del tiempo. De la ec. 2 , se deduce lo siguiente:

a. Sí $R^{\prime} C>0$ (la razón circulante marginal es mayor que cero), entonces es creciente.

b. Sí $R^{\prime} C^{\prime}=0$ (la razón circulante marginal es igual a cero), entonces hay un punto crítico, que puede ser un valor máximo o un valor mínimo.

c. Sí $R^{\prime} C^{<} 0$ (la razón circulante marginal es menor que cero), entonces es decreciente.

Otro modelo matemático que se puede aplicar a las cuentas de la contabilidad, cuando su comportamiento no es variable, corresponde a la teoría de conjuntos como se describe en 7.4

\subsection{NOCIÓN Y DEFINICIÓN DE CONJUNTO EN MATEMÁTICAS.}

En general se define como una colección de objetos, sin embargo, dadas las ambigüedades alrededor de las posibles contradicciones con la definición anterior es posible también comparar la noción de conjunto desde un concepto más general llamado clase, la cual también es una colección de objetos, así una clase también es una colección de objetos que pueden ser conjuntos. Para Cantor (1845-1918) matemático ruso, quien definió rigurosamente el concepto de conjunto, dijo que: un conjunto quedaba definido si era posible describir completamente sus elementos, es decir, de acuerdo con sus características por comprensión o nombrar 
todos sus elementos uno a uno, por extensión. Los elementos de un conjunto por lo general se encierran entre llaves, por ejemplo, sea el conjunto de los números dígitos por extensión, $D=\{0,1,2,3,4,5,6,7,8,9\}$ o por comprensión: $D=$ $\{x / x \notin 0 \leq N \leq 9\}$.

La teoría de conjuntos está dotada de símbolos como:

$\notin$ : pertenencia, significa que algún elemento pertenece a un conjunto dado, $x \notin A$ (el elemento $x$ pertenece al conjunto $\mathrm{A}$. $\mathrm{Si}$, por el contrario, un elemento no pertenece a un conjunto dado, se notará con el símbolo $\notin$ por ejemplo, $1.5 \notin \mathrm{N}$ (1.5 no pertenece al conjunto de los números naturales $\mathrm{N}$ ).

Los símbolos lógicos, tomados de la lógica de predicados: $\neg$ (no: negación), ^ (y: conjunción), $\checkmark$ (o: disyunción), $\rightarrow$ (entonces: implicación), $\leftrightarrow$ (si y sólo si: equivalencia), $\forall$ (para todo: cuantificador universal) y $\exists$ (Existe: cuantificador existencial) y (,) (paréntesis).

Además, los conjuntos o clases se pueden operar, a través de la unión, intersección o diferencia (como operaciones propias entre ellos como conjuntos).

1. Unión: $A \cup B=\{x /(x \notin A) \vee(x \notin B)\}$ : A unión $B$ está formado por los elementos que están en $A$ o están en $B$. Además, se puede verificar que: $\forall \mathrm{x}(\mathrm{x} \notin A \cup B \leftrightarrow(\mathrm{x} \notin A) \vee(x \notin B))$.

2. Intersección: $A \cap B=\{x /(x \notin A) \wedge(x \notin B)\}$ : $A$ intersecado con $B$ está formado por los elementos que están en $A$ y que están en B. Se puede verificar que: $\forall x(x \notin A \cap B \leftrightarrow(x$ $\notin A) \wedge(x \notin B))$.

3. Diferencia: $A-B=\{x /(x \notin A) \wedge(x \notin B)\}$ : A menos $B$ está formado por todos los elementos que están en $A$ pero no en $B$. Se puede verificar que: $\forall x(x \notin A-B \leftrightarrow(x \notin A) \neg(x \notin B))$.
A partir de lo anterior es posible decir que el Balance General es una clase o conjunto, formado por elementos llamados cuentas reales, las cuales corresponden a los Activos: A, Pasivos: $P$ y Patrimonio neto: $P n$, además se establece que los elementos de los conjuntos anteriores son las cuentas, las cuales de acuerdo con sus características pertenecerán a uno y sólo uno de ellos. Así, por ejemplo, $A=$ activos corrientes, activos no corrientes\}; es posible también decir entonces que el conjunto de los activos está conformado por la unión entre los subconjuntos \{activos corrientes\} $\cup$ \{no corrientes\}, de forma similar el conjunto de los pasivos, $\mathrm{P}=$ \{pasivos corrientes, pasivos no corrientes\}. El conjunto de patrimonio $\mathrm{Pn}=$ \{capital contable, reservas, resultados acumulados, resultados de ejercicio, otros componentes del patrimonio\}. Sin embargo, el patrimonio también es equivalente a la diferencia entre los conjuntos de activos menos los pasivos (A-P). En general con las cuentas anteriores se pueden usar los símbolos lógicos, así como también se cumplen las operaciones que se describen entre conjuntos.

\section{PROPUESTA DE MODELACIÓN CONTABLE CUANTITATIVA.}

En este apartado se tendrán en cuenta algunos aspectos recopilados en la primera fase de investigación, surgidos de la revisión documental hecha sobre modelación matemática en contabilidad. La contabilidad como ciencia se beneficia a través del uso de modelos matemáticos, así como se ha podido evidenciar en otras ciencias. En este sentido es importante el estudio de las variables contables desde las matemáticas, para darle rigor científico y posibilitar acuerdos académicos. (Cano,2008, pág. 119).

"El desarrollo de modelos matemáticos ofrece enormes posibilidades de avance científico para la contabilidad como ha sucedido con las demás disciplinas y ciencias. Estudiar la 
expresión de las variables contables en términos matemáticos es una exigencia ineludible y necesaria si se quiere dar a la contabilidad un carácter más académico, actual y científico." (Cano, $2008: 119)$.

La variable es un concepto fundamental de las matemáticas que permite estudiar algunos fenómenos y procesos de variación tanto en las ciencias naturales como sociales, indagando particularmente en; el qué varía, cómo varía y por qué varía. Así pues, tanto un fenómeno natural (la lluvia, el calentamiento global, movimiento telúrico, huracanes, ...) como un proceso social (contabilidad, ventas, producción, sociedad, cultura, ...) son estudiados por expertos que indagan sobre ellos a partir del interés por entenderlos, explicarlos, clasificarlos y predecirlos o anticiparlos desde una realidad particular.

Todo proceso de variación genera cambios a partir de las interacciones entre las variables intervinientes que se pueden inferir o abstraer de una realidad, a través de relaciones de dependencia $\circ$ correlación que permiten clasificar a las variables como independientes y dependientes. Una variable es independiente si y solo si, los valores que toma en un intervalo dado son arbitrarios y no dependen de ninguna otra variable interviniente en el suceso o situación, en caso contrario será dependiente, por ejemplo, la ubicación de un inmueble y su precio, la ubicación es una variable independiente respecto al precio y su precio depende de la ubicación; así como de otras variables como los acabados, el área de construcción,..., las cuales serán variables independientes también respecto al precio del inmueble, que además no generan dependencia entre sí, pero todas ellas tienen una correlación respecto al valor del inmueble.

García Casella (2001), en el trabajo de investigación llamado Elementos para una teoría general de la contabilidad, propone algunas situaciones problémicas que según él deben ser tenidas en cuenta para su estudio. Entre ellas, resalta la importancia de la medición y la modelación contable que da soporte a la contabilidad como ciencia de naturaleza cuantitativa. La contabilidad, dice también Casella (2001); que es una ciencia de naturaleza fáctica, cultural y aplicada cuya finalidad es la de organizar, dar explicación y determinar las normas en tareas de descripción primordialmente de índole cuantitativo entre otras.

Es posible a partir de lo anterior proponer explicaciones que describan situaciones contables en las que las variables presentes en ella generen cambios como los dados en la fig. 1; la cual representa un proceso variacional cíclico en un balance general, lo cual significa que se repite con alguna periodicidad y que por lo tanto puede ser modelado a partir de una interpretación de la realidad en la que se ubica.

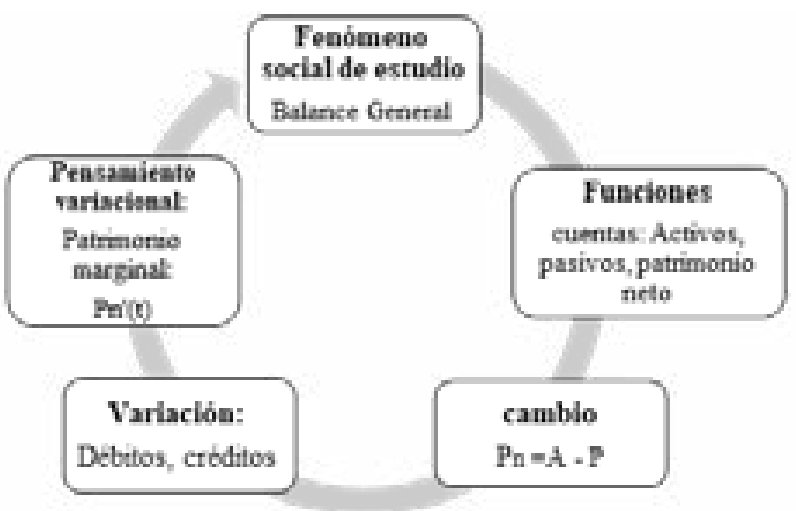

Fig. 1. Funciones del tiempo en contabilidad.

Fuente: propia.

En el estudio de un fenómeno o proceso de variación contable, fig.1, es importante analizar el cambio que se produce por una transformación cuantitativa de las funciones intervinientes en ellos, dichos cambios al ser cuantitativos dan lugar a valores numéricos, cuyos registros se convierten en datos que almacenan información contable del fenómeno o proceso en cuestión importante para conjeturar, predecir y anticipar 
situaciones posibles por ser inherentes a los procesos de variación. Por ejemplo, en un balance general en el que las cuentas pertenecen a tres conjuntos diferentes pero relacionados entre sí, cuya naturaleza es variable como la de los activos, pasivos y patrimonio neto es fácil advertir que el cambio de valor en el conjunto de los activos o pasivos genera cambios en el del patrimonio neto (capital contable).

Este proceso variacional se puede modelar matemáticamente dado que sus funciones que varían en el tiempo, establecen relaciones tanto de dependencia como de temporalidad periódica y su modelo es conocido por la ecuación, $P_{n}=A-P_{n}$, donde patrimonio neto, A: activos y $P$ : pasivos; puede medir con valores fijos o variables (intervalo de valores posibles) para las tres cuentas involucradas en el balance general que determinan la ecuación contable de patrimonio neto. En este caso, el proceso de variación puede dar lugar a una razón de cambio como en la [ec.3], porque es posible establecer una relación en el tiempo (dos momentos $t$ y) entre las funciones contables del balance, de la siguiente forma:

$A_{1}=A+\Delta A ; \quad P_{1}=P+\Delta P$ y $P_{n 1}=P_{n}+\Delta P_{n} . \quad[$ ec. 3].

En este caso, en un período de tiempo dado los conjuntos de los activos $A$, pasivos $P$ y patrimonio neto pueden aumentar o disminuir un valor, significa entonces la diferencia que se presenta en un conjunto contable al variar en un período de tiempo determinado, donde . Esta relación de las funciones $\mathrm{A}$ y $\mathrm{P}$, pueden generar dos situaciones:

$>$ La primera que los activos y los pasivos se mantengan iguales en un período de tiempo, como en la ec.4.

$$
A=A_{1} \text { y } P=P_{1}[\text { ec. } 4]
$$

Según la Ec. 4 , se cumple que $\Delta \boldsymbol{A}=\boldsymbol{A}$ y que $\Delta \boldsymbol{P}=\boldsymbol{P}$, lo cual significa que no hay variación en el tiempo de las funciones contables, es decir que se mantienen constantes, luego el patrimonio neto tampoco cambia: $\Delta \boldsymbol{P}_{\boldsymbol{n}}=\boldsymbol{P}_{\boldsymbol{n}}$

La segunda que tanto los activos como los pasivos cambien en el tiempo, es decir que: $\boldsymbol{A} \neq \boldsymbol{A}_{\mathbf{1}}$ y $\boldsymbol{P} \neq \boldsymbol{P}_{\mathbf{1}}$. En este segundo caso, se puede presentar la siguiente relación, dada en la ec.5.

$$
\frac{A_{1}-A}{P_{1}-P}=\frac{\Delta A}{\Delta P} \quad(\text { Tasa de Variación Media: TVM })
$$

Esta relación, representa la razón de cambio de activos respecto a los pasivos en un tiempo determinado. A partir de ella se pueden dar las siguientes razones de cambio o TVM,

a. $\frac{\Delta A}{\Delta P}<\mathbf{1}$, es decir que los pasivos son mayores que los activos, a partir de lo cual el patrimonio neto será negativo, significando con ello decrecimiento o pérdidas de patrimonio, que de no ser tratada con atención y cuidado podría acarrear la quiebra o desaparición de una organización o empresa inclusive.

b. $\frac{\Delta A}{\Delta P}=1$, en este caso los activos son iguales a los pasivos, hay equilibrio entre pasivos y activos no sobran las utilidades o activos que respalden, tampoco hay pérdidas y por tanto el patrimonio neto se mantiene constante, pudiendo ser cero.

c. $\frac{\Delta A}{\Delta P}>1$, se da cuando los activos son mayores que los pasivos, con lo cual se garantiza un crecimiento patrimonial temporal, siendo importante aclarar que se puede presentar un "capital ocioso" (en el caso de activos y pasivos circulantes) si el valor de la razón es muy grande, lo cual puede interpretarse como una mala gestión con la inversión del capital sobrante que podría beneficiar a la entidad o empresa a futuro.

Otras relaciones de temporalidad $(\Delta$ : signo matemático que determina cambio en el tiempo), donde $\boldsymbol{t} \neq \boldsymbol{t}_{\mathbf{1}}$ a partir de la ecuación de patrimonio que pueden generar variación en 
procesos contables de una entidad o empresa, se presentan en las ecuaciones 5,6 y 7 :

$$
\frac{P_{n}}{\Delta \boldsymbol{P}_{n}}=\frac{\boldsymbol{A}-\boldsymbol{P}}{\Delta \boldsymbol{A}-\Delta \boldsymbol{P}} \quad \operatorname{con} \Delta \boldsymbol{P}_{n} \neq \mathbf{0} \quad[\text { ec.6] }
$$

La relación presentada en la ec.6, permite medir el crecimiento del patrimonio neto cuando $P_{n}>\Delta P_{n}$, decrecimiento cuando se cumple que $P_{n}<\Delta P_{n}$ en un tiempo ( $\mathrm{t}$ ) determinado de medición, además la relación establecida por la ecuación [ec.6] es de equivalencia donde la razón de cambio patrimonial o TVM, debe garantizar que sea diferente de cero, porque de lo contrario la razón de cambio (TVM) no estaría definida.

A partir de la ec.6, se puede plantear otra relación para hacer una medición del patrimonio neto $P_{n}$ como una tasa de variación instantánea o razón de cambio instantánea como se presenta en la ecuación 7.

$$
\frac{P_{n}}{\Delta P_{n}}=\frac{A-P}{\Delta P_{n}} \quad[\text { ec. } 7]
$$

para este caso ec.7, es posible asumir que $P$ $=A+\Delta A$ (pasivos iguales a los activos más un incremento en activos).

1. La razón de cambio [ec.7] mide la tasa de variación media del patrimonio neto (TVM) para cualquier diferencia de tiempo distinta de cero $(\Delta t \neq 0)$.

2. La razón de cambio [ec.7] será una medición instantánea de patrimonio neto si la diferencia de patrimonios tiende a ser cero en un período de tiempo fijo ( $\Delta P_{n} \rightarrow$ $0)$ es decir se acerca a cero, pero nunca llega a ser cero, lo cual genera una nueva relación llamada la derivada del patrimonio neto patrimonio neto marginal; que mide la velocidad con la que cambian los activos y los pasivos en un tiempo determinado del balance general.
3. La razón de cambio patrimonial puede expresarse como una función del tiempo y se podría notar como:

$P_{n}(A)=P_{n}(t)=A(t)-[A+\Delta A](t)$, lo cual significa que el patrimonio es una función del tiempo igual a los activos menos los pasivos los cuales también dependen de los activos $A$ y de su incremento $\Delta A$ en un tiempo t.

4. La función patrimonial puede expresarse como una razón de cambio media en el tiempo, a través del cociente incremental:

$$
\boldsymbol{P}_{n}(\boldsymbol{A}) \boldsymbol{P}_{n}(\boldsymbol{A})=\frac{\boldsymbol{P}_{n}(\boldsymbol{A}+\Delta A)-\boldsymbol{P}_{n}(\boldsymbol{A})}{\Delta \boldsymbol{A}} . \quad[\mathrm{ec} .8]
$$

Si en la anterior ecuación $8, \Delta \mathrm{A} \rightarrow 0(\Delta \mathrm{A}$ tiende a ser cero) y se garantiza que la función patrimoniales continua en todo instante $\mathrm{t}$ (de un período determinado) se puede hallar su primera derivada, como en la ec.9.

$$
P_{n}^{\prime}(A)=\lim _{\Delta A \rightarrow 0} \frac{P_{n}(A+\Delta A)-P_{n}(A)}{\Delta A} . \quad[\text { ec.9] }
$$

A partir de la ec.9, es posible determinar:

a. Velocidad de cambio del patrimonio en el tiempo o patrimonio marginal.

b. Razón de cambio instantánea de patrimonios para un tiempo determinado $\left(t=t_{0}\right)$.

c. También es posible determinar los activos marginales, haciendo que la ecuación contable sea de la forma: $A=P+P n$. y a partir de ello resolver para la cuenta de activos $A$ como en la ec. 8 y usar la información como un evento conjuntista similar al propuesto en la fig. 2. 


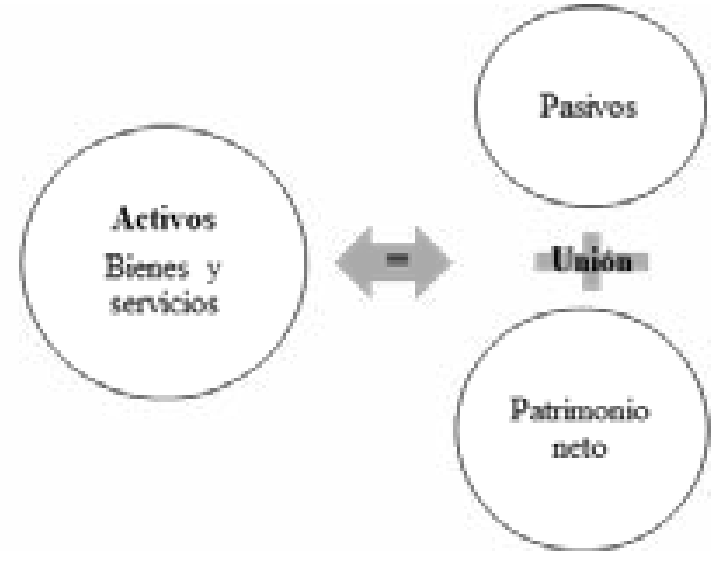

Fig. 2, Balance General como conjunto

Fuente: propia

En la fig. 2., se establece una relación de variables que determinan la naturaleza de un balance general, el cual en un tiempo determinado muestra un estado financiero de una empresa u organización determinada. En la relación se aprecia que los activos, los pasivos y el patrimonio son conjuntos integrados por cuentas y por lo tanto se operan como tal, es así que al estudiar la procedencia de los recursos en una organización se deben unir las cuentas de los conjuntos que representan los pasivos con las del patrimonio, en tanto que los activos como conjunto de cuentas corresponden al resultado de dicha unión. Lo anterior llama la atención en la manera particular en la que usualmente se suman las cuentas involucradas en la ecuación de patrimonio neto: $A=P+P n$ (los activos son iguales a la suma de pasivos con el patrimonio ${ }^{9}$ ), no es que esté incorrecta, es solo que es limitada, porque tanto los activos como los pasivos son en realidad conjuntos de cuentas, por ejemplo, los activos corrientes y los activos no corrientes son subconjuntos de los activos; así como los pasivos corrientes y los pasivos no corrientes lo son de los pasivos. Es importante observar también que, en un estado

$9 \quad$ La suma corresponde al valor numérico de las cantidades finales en un estado financiero, es decir al número cardinal del resultado total acumulado en el conjunto de cada cuenta. financiero determinado, tanto los pasivos como el patrimonio son subconjuntos de los activos.

Teniendo en cuenta lo anterior toda empresa u organización social para poder funcionar necesita de los conjuntos de activos y de patrimonio o capital de trabajo para poder operar, puesto que por lo general la entidad debe asumir compromisos financieros para poder generar activos, dado que los recursos no se pueden crear por sí mismos, sino que necesitan de una fuente para ello; por lo tanto, se diferencia empresa u organización social de quien la financia, dado que la primera para poder trabajar o funcionar requiere de la segunda o contrapartida (financiación).

Otro aspecto importante para una empresa u organización, lo constituye la manera de financiarse, la cual puede ser de dos formas: 1. La financiación resulta del aporte de los socios, accionistas o dueños de la organización o entidad, de donde los activos son iguales al patrimonio. En este caso los activos corresponden a los bienes y al capital de trabajo de la entidad y el patrimonio corresponde a los recursos propios de los dueños, socios u accionistas; quienes los suministraron a la organización o entidad con el fin de permitir su desarrollo en activos y capital de trabajo. 2. En el caso de que los dueños, accionistas o socios no dispongan de recursos necesarios para financiar los activos y el capital de trabajo de la empresa o entidad para su funcionamiento, entonces se requiere de los pasivos que corresponden a la financiación de terceros, luego en este caso los activos son financiados por los dueños a través del patrimonio, una parte y la otra restante por terceros o pasivos. En este sentido la ecuación patrimonial corresponde a la equivalencia entre lo que se tiene, conjunto de activos con lo que se debe, conjuntos de pasivos y patrimonio; la ecuación de patrimonio neto corresponde a un fenómeno variacional en un período determinado 
que permite medir los hechos económicos de una empresa o entidad.

De otra parte, es importante resaltar en la relación como conjunto de entre las cuentas de patrimonio neto $(P n)$, activos $(A)$ y pasivos $(P)$. [ec.10]

\section{$\boldsymbol{A}=\boldsymbol{P}+\boldsymbol{P n} \quad[$ ec. 10$]$}

De la ec.10 se puede afirmar que las cuentas relacionadas pueden establecer interacciones diferentes como las siguientes:

a. (A), aumenta cuando los pasivos y el patrimonio se incrementan, porque para generar los activos se requiere de la financiación de terceros, por ejemplo, el aporte en bienes que hace un socio, propietario o accionista generan los activos de la empresa y cuando se toma un crédito bancario, este genera dinero en efectivo que corresponde a los activos.

b. (A), disminuye si los pasivos o el patrimonio disminuyen también, por ejemplo, cuando una empresa o entidad cancela un crédito con un banco, debe hacerse con dinero en efectivo que permita pagar la obligación con terceros, disminuyendo los activos para ello. De la misma forma sucede con el patrimonio, por ejemplo, al distribuir las utilidades generadas en una empresa o entidad, los pagos generados disminuyen los activos también.

Ahora, si la relación de equivalencia anterior ec. 10, toma la forma de la ec.11.

$P n=A-P \quad[$ ec. 11$]$

entonces es posible deducir a partir de la ec.11, que:

a. El patrimonio $(P n)$ se aumente al disminuir los pasivos, por ejemplo, al capitalizar un pasivo, a través del pago en acciones. De la misma forma, se puede disminuir el patrimonio aumentando los pasivos, por ejemplo, al pagar las utilidades generadas por una empresa con un sobregiro bancario. 2. Otra forma de afectar el conjunto del patrimonio neto es a través de la diferencia entre los ingresos, costos y gastos; por ejemplo, si los ingresos son mayores que los costos y gastos entonces hay utilidades, en caso contrario hay pérdidas. Las utilidades corresponden a un crédito en la cuenta del conjunto del patrimonio y sus pérdidas corresponden a un débito a la cuenta del conjunto de patrimonio, porque como ya es conocido las cuentas del conjunto del patrimonio son de naturaleza crédito, por lo tanto, los débitos disminuyen el valor del patrimonio neto.

\section{CONCLUSIONES}

A pesar de haber realizado un estudio documental sobre el balance general y sus cuentas respectivas, apoyado en referencias bibliográficas surgidas del interés histórico de algunos investigadores en torno a la modelación matemática como apoyo a la contabilidad axiomática, así mismo como soporte de los conceptos que de la contabilidad se han trabajado en este artículo; es importante aclarar que no se ha realizado una fase de experimentación en la práctica contable y que por lo tanto la modelación expuesta en este trabajo es eminentemente desde las matemáticas apoyada eso sí, en la literatura y en conceptos de la contabilidad.

La puesta en práctica de los resultados expuestos en este artículo y que surgen de la investigación reportada será producto de otra investigación interdisciplinar con un grupo de investigación contable, cuyo trabajo de campo en el área contable como investigación experimental permita su verificación y ajustes.

Las cuentas propuestas en este artículo, desde la literatura de la contabilidad (teorías e investigaciones consultadas) se comportan como funciones matemáticas y por ello la motivación de indagar otras interacciones con ellas que además las matemáticas posibilitan en los procesos variacionales. 


\section{REFERENCIAS BIBLIOGRAFICAS}

Balzer, W. \& Mattessich, R. (1991): An axiomatic basis of accounting: a structuralist reconstruction. Theory and decision, vol. 30. Netherlands: Kluwer Academic Publisher.

Beaver, W., H. (1996): Directions in accounting research: NEAR and FAR. Accounting Horizons. Traducción de Carlos Luis García Casella, vol. $10(2)$.

Bry, X. \& Casta, J., F. (1995): Measurement, Imprecision and uncertainty in financial accounting: can double entry be understood with fuzzy numbers? Fuzzy Economy Review, Number 0 , november.

Cano, M., A., M. (2008): Preludio histórico de la Contabilidad y su analogía directa con la Modelación Matemática. Revista Universo Contábil, vol. 4, núm. 4 octubre-diciembre, pp. 112-125. ISSN: 1809-3337.

Cano M., A., M.; Zapata M., Á.; Ramírez H., L., M. (2005): Preliminares sobre el desarrollo histórico de la contabilidad y su relación con los modelos matemáticos. Revista Contaduría Universidad de Antioquia, n. 46, p. 14-46, juliodiciembre.

Chaves, O., A. (1990): Tratamiento contable del patrimonio neto, análisis de los aspectos doctrinarios, técnicos y legales y su adecuación a la realidad argentina, Buenos Aires: Universidad de Buenos Aires, Facultad de Ciencias Económicas. Tesis doctoral (Biblioteca, Alfredo L. Palacios).

Correa, G., J., A. (2005): De la partida doble al análisis financiero. Contaduría, universidad de Antioquia, 46 (1) pp.169-194.

Churruca, A., E. (1982): Contabilidad analítica matricial para un sistema de fabricación intermitente. Revista Española de Financiación y Contabilidad, REFC, XI (38-39), 515-554.
Disponible en: https://dialnet.unirioja.es/ descarga/ articulo/2481859.pdf

Fowler, N., E. (2011): Cuestiones Contables Fundamentales. Pp., 299-314, 5a edición. Editorial La Ley. ISBN: 978-987-03-1896-5.

Fowler, N., E. (2008): Cuestiones Contables Fundamentales. 4a edición. Editorial La Ley.

García C., C., L. (2001): Elementos para una teoría general de la contabilidad. Buenos Aires Argentina: Editorial La Ley, $1^{\text {a }}$ Edición, 2da quincena de junio de 2001.

García C., C., L. (2002). Hacia un incremento de la modelización contable. Buenos Aires Argentina: Ediciones Cooperativas.

García C., C., L.; Rodríguez De R., M. Del C. (2001): Elementos para una Teoría General de la Contabilidad. Buenos Aires Argentina: Ed. La Ley.

García C., C., L. $\quad(1997$ a): Enfoque Multiparadigmático de la Contabilidad: Modelos, Sistemas y Prácticas deducibles para diversos contextos. Buenos Aires Argentina: CONICET (2 tomos) Edición Limitada.

García C., C., L. (2005): El dominio del discurso contable en la Contabilidad social; proyecto UBAC y T EO 24, 2004 a 2007. Buenos Aires Argentina: Ediciones Cooperativas.

García C., C., L. (1997 b): Naturaleza de la Contabilidad. S.I. Contables, Ed. Contabilidad y Auditoría, mayo, año 3(5), pp.13, 12-37.

García, G., M. (1975): El paradigma de la partida doble en la ciencia contable: Análisis crítico de sus versiones en los modelos de la contabilidad matricial y multidimensional frente al modelo convencional. Revista española de financiación y contabilidad V. IV (12 y 13), pp. 341-364. 
Griffin. CH., N. \& Thomas H., W. (1987): Un análisis comparativo de la contabilidad y las matemáticas. México: Editorial Diana, pp. 333341.

Guajardo C., G., \& Andrade De G. (2008): Contabilidad financiera. México: McGraw Hill/ Interamericana editores SA de CV.

Hatzacorsian H., V. (2003): Fundamentos de contabilidad. México: International Thomson Editores S. A. de C.V.

ljiri, Y. (1967): The Foundations of Accounting Measurement: A. Mathematical, Economic, and Behavioral Inquiry. Englewood Cliffs, New Jersey: Prentice-Hall.

Kennedy, R. D., \& Mcmullen, S., Y. (1971): Estados financieros. Forma, análisis e interpretación. Homewood, Illinois, E.U.A.: Unión Tipográfica Editorial Hispanoamericana.

Lopes De Sá, A. (2002 a): Origen y evolución del conocimiento contable. Enciclopedia de contabilidad, Bogotá, Panamericana.

Lopes De Sá, A. (2002 b): Luca Pacioli: hombre del renacimiento. Revista Legis del contador $\mathrm{N}^{\circ}$ 10, Editorial Legis, pp. 82-97.

Lopes De Sá, A. (2003): Bases de las escuelas europea y norteamericana frente a la cultura contable y la propuesta Neopatrimonialista. Revista Internacional Legis de contabilidad y Auditoría $\mathrm{N}^{\circ} 13$, editorial Legis, pp. 69-92.

Lopes De Sá, A. (2007): Doctrinas, escuelas y nuevas razones de entendimiento para la ciencia contable, en revista Contabilidad \& Auditoría de Legis, $\mathrm{N}^{\circ} 30$, abril-junio de 2008.

Mattessich, R. (2002): Contabilidad y métodos analíticos. Medición y proyección del ingreso y la riqueza en la microeconomía y macroeconomía, Traducción García Casella y Rodríguez de Ramirez. Buenos Aires: Ed. La Ley.
Mattessich, R. [1964-2002]: Contabilidad y Métodos Analíticos Medición y proyección del Ingreso y la Riqueza en la Microeconomía y en la Macroeconomía, Editorial La Ley S.A. Buenos Aires, Argentina. ISBN 950-527-731-8.

Mattessich, R. [1958]: Mathematical models in business accounting. Accounting Review. Source: The Accounting Review, Vol. 33, No. 3 (jul, 1958), pp. 472-481 Published by: American Accounting Association Stable. Recuperado el 03 de septiembre de 2017 de URL: http://www. jstor.org/stable/Accessed:

MEN (1998): Serie Lineamientos Curriculares Matemáticas. Recuperado el 12 de febrero de 2019 de https://www.mineducacion.gov.co/1621/ articles-89869 archivo pdf9.pdf

MEN (2003): Estándares Básicos de competencias en Matemáticas. Recuperado el 12 de febrero de 2019 de https://www. mineducacion.gov.co/1621/articles-89869 archivo_pdf9.pdf

Moreno, F., J. (2002): Contabilidad Superior. México: Instituto Mexicano de Contadores Públicos AC.

Prieto, A. (2009): Contabilidad Básica enfocada a Normas de Información Financiera. México: Editorial Limusa SA de CV.

Pyle, W., W., J., A. \& Larson, K., D. (1981): Principios fundamentales de contabilidad. México: Compañía editorial continental S.A.

Ramírez, R., E. (2017): Métricas matemáticas y modelación contable, editorial REDIPE-Dialnet, (agosto). Vol 6(8).

Ramirez, R., E. \& Avellaneda, B., C., A. (2016): Episteme de la ecuación de patrimonios. Criterio Libre, vol 14(25). (julio-diciembre), pp. 33-45. 
Ramirez, R., E. (2016): El cálculo en la formación de competencias profesionales de contadores, Editorial REDIPE - Dialnet, Vol 5(8), pp. 61-75, ISNN 2266-1536.

Romero López, Á. J. (2002): Principios de contabilidad. México: Mc Graw Hill.

Rojas Y Aguilar, H. (1996): Apuntes de análisis e interpretación de los estados financieros. México: Instituto Politécnico Nacional.

Tua, P., J. (1995): Lectura de Teoría e investigación Contable. Ediciones Gráficas Ltda. octubre-diciembre, Medellín, Colombia.

Tua, P., J. (2004): Evolución y situación actual del pensamiento contable. Revista internacional legis de contabilidad y auditoría, 20, octubrediciembre, pp. 129-144.

Tua, P., J. (1993): La investigación en contabilidad: una reflexión personal. Ponencia II Encuentro de Investigación Contable, Fundación para la Investigación y Desarrollo de la Ciencia Contable. Bogotá, mayo de 1992.

Tua, P., J. (1988): Evolución del concepto de contabilidad a través de sus definiciones. Contaduría Universidad de Antioquia, pp.13, 9-74. 